\title{
Image-Based Relighting of Moving Objects with Specular Reflection
}

\author{
Hanhoon Park ${ }^{1}$, Jong-Il Park ${ }^{1}$, and Sang Hwa Lee ${ }^{2}$ \\ 1 Division of Electrical and Computer Engineering, Hanyang University, Seoul, Korea \\ \{hanuni, jipark\}@mr.hanyang.ac.kr \\ 2 Department of Electrical Engineering, Seoul National University, Seoul, Korea \\ lsh@ipl.snu.ac.kr
}

\begin{abstract}
In the fields of Augmented Reality (AR) and Virtual Reality (VR), inserting an object into a scene (real or virtual) requires proper matching of their lighting conditions. If not, the resulting image may look unnatural. In particular, it is important to describe the position and shape of specular reflection accurately if the object has specular reflection. In this paper, we propose an approach to relighting a moving object based on the separation of specular and diffuse reflection. To relight an object, two or more images taken under the condition that the position of the object is fixed but the lighting condition is different, we call synchronized images, are required. However, it is impossible to obtain such images in case of a moving object. Therefore, we propose a method that computationally obtains the synchronized images using the consecutive fields of a controlled video sequence containing a moving object. For example, if the virtual $(n+1)-t h$ field is interpolated from $n-t h$ field and $(n+2)-t h$ field using the motion compensation technique, both the virtual $(n+1)-t h$ field and the real $(n+1)-t h$ field have the condition that the position of the object is fixed. If the virtual and real image have different lighting condition, the method applied to static object is applicable to moving object as it is. After the specular and diffuse reflection are separted, the relit image is synthesized using the linear interpolation and morphing technique. The experimental results of applying the proposed method to real and synthetic images are given. We verify the effectiveness of the proposed method by comparing the resulting image with a ground-truth image.
\end{abstract}

\section{Introduction}

Matching the lighting condition of the involved images is a necessary step in synthesizing photorealistic images from different image sources. In particular, it is important but hard to handle the specular reflection, which is necessary to render an object realistically. For convenience, most of computer graphics algorithms have assumed that the object has no specular reflection (i.e. the surface is Lambertian) 1/2/34. Obviously, this assumption does not fully satisfy the reality requirements in computer graphics. Recently, several approaches associated with image based relighting (IBL) have been reported to handle the 
specular reflection independently by separating specular reflection from diffuse reflection. Many approaches for separating specular and diffuse reflection have been proposed [56/7814] and successfully applied to relighting static objects 9 .

There have been several approaches for changing the lighting condition of an image 11 12 13. They require capturing numerous images using a number of light sources and focus on relighting static objects. Recently, an efficient approach for relighting a static object in which only two images are required was proposed 9. To relight a static object, two or more images that are taken in the condition that the position of the object is fixed but the lighting condition is different are required [9]. By comparing the values of the corresponding pixels on the images with each other, specular and diffuse reflection are separated and used for relighting the object independently.

This paper is an extension of [9] toward coping with a moving object. Notice that the algorithm for relighting a static object can be used for relighting a moving object as it is if we can obtain a pair of synchronized images with different lighting condition from a video sequence containing a moving object, Therefore, we propose a method that computes a pair of synchronized images from the consecutive fields of a controlled video sequence of a moving object, where the lighting conditions of even/odd field are alternating. The synchronized images are obtained by applying a motion-compensated interpolation algorithm to the controlled video sequence.

\section{Image-Based Relighting Based on the Separation of Specular and Diffuse Reflection}

In this section, we briefly explain the process of relighting a static object based on the separation of specular and diffuse reflection. Given two images on which a static object is taken in the different lighting condition, the specular and diffuse reflection are separated. To relight the static object, a linear interpolation algorithm and a morphing algorithm are applied to the resulting specular and diffuse reflection, separately. The shaded region in Figure 4 depicts the overall process for relighing a static object.

\subsection{Separation of Specular and Diffuse Reflection}

Specular reflection is an important factor for describing an object realistically but a troublesome in the field of computer vision. Most of computer vision algorithms have assumed that the object has no specular reflection. To handle specular reflection effectively, it is necessary to separate specular reflection from diffuse reflection. In the literature, the algorithms for separating specular and diffuse reflection are mainly divided into two categories: color-based [5614]; polarization-based 78 . Both kinds of algorithms have shown satisfactory results but the former requires lots of images for processing, the latter has a difficulty in obtaining suitably polarized images. In this paper, the more effective method are used, which is a color-based method but requires only a few images [9]. The 


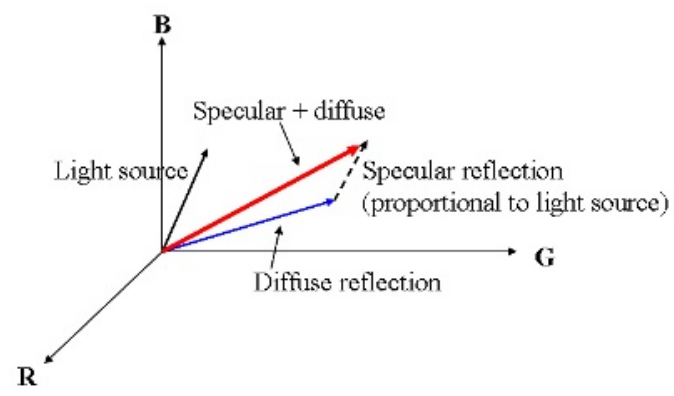

Fig. 1. Property of specular and diffuse reflection

method is simpler and more effective than the method proposed by Lin and Shum [6] which is based on complicated spectrum analysis.

Two images are used on which an object is taken in the different lighting condition. Given image $A$ and $B$, the pixel values of each image are denoted by the sum of specluar and diffuse reflection [14. Then, the specular and diffuse reflection can be represented in RGB color space as shown in Figure 1 because the intensity of specular reflection is proportional to the intensity of light source [10. If the intensity of an arbitrary pixel on image $B$ is lower than that of the corresponding pixel on image $A$, it is regarded that the pixel has no specular reflection while the correspoding pixel may have specular reflection. Given a light source as $\boldsymbol{E}_{\boldsymbol{k}}(k=r, g, b)$, the specular reflection is represented as $t \boldsymbol{E}_{\boldsymbol{k}}(t \geq 0)$. the specular reflection can be separated from the diffuse reflection using Eq. (1) which is derived from the fact that the RGB values of diffuse reflection are changed while the ratio among them is preserved when the lighting condition is changed [10].

$$
\frac{r\left(I_{A}\right)-t \boldsymbol{E}_{\boldsymbol{r}}}{r\left(I_{B}\right)}=\frac{g\left(I_{A}\right)-t \boldsymbol{E}_{\boldsymbol{g}}}{g\left(I_{B}\right)}=\frac{b\left(I_{A}\right)-t \boldsymbol{E}_{\boldsymbol{b}}}{b\left(I_{B}\right)}
$$

where $\left.\mathrm{r}^{*}\right), \mathrm{g}\left({ }^{*}\right), \mathrm{b}\left({ }^{*}\right)$ : the value of red, green, and blue.

\subsection{Synthesis of Virtual Specular and Diffuse Reflection}

Diffuse reflection by a virtual-light-source $C$ can be computed by linearly interpolating diffuse reflection by a real-light-source $A$ and diffuse reflection by a real-light-source $B$. Image morphing is used to synthesize the specular reflection by a virtual-light-source. In particular, voxel-based morphing is used because it can be consistently applied to a whole image and thus easy-to-use compared with polygon-based one which requires to discriminate the object area from another area and approximate it to a polygon.

Given two objects which are represented by its center and size at onedimensional space, the center and size of the intermediate object can be computed by linearly interpolating those of the objects. Two-dimensional image morphing is achieved by applying the one-dimensional morphing to each row 
of an image, independently. Specular reflection by a virtual-light-source is computed by applying the two-dimensional image morphing algorithm to specular reflections by two real-light-sources which have the different position from each other. The morphing algorithm does not work if the height of target objects are different from each other. Therefore, after computing the minimum-boundingbox including specular region and normalizing the size of the box to a fixed size, the morphing algorithm is applied to the normalized region. The transformed specular region is located at the virtual image by applying linear interpolation algorithm to the center, width, and height of the specular area by two real-lightsources, respectively [9].

\section{Computing Synchronized Images for Moving Object}

In this section, we explain how to relight moving objects. The method of relighting a moving object is the same as that for a static object. However, since we cannot obtain a pair of images required for relighting a moving object from a video sequence directly, we compute the required images from a controlled video sequence of a moving object. Different lighting conditions are applied to the odd-field and even-field of an interlaced video sequence alternatively using a controlled strobe light. Then, the required images are synthesized using a temporal interpolation algorithm.

The motion of an object between the fields is assumed to be small because the temporal gap between the fields of video sequences is very small. Thus, if the virtual $(n+1)-t h$ field is interpolated from $n-t h$ field and $(n+2)-t h$ field using the motion compensation technique, the virtual $(n+1)-t h$ field and the real $(n+1)-t h$ field satisfy the condition that the position and shape of the object is fixed. Because the virtual and real images have different lighting

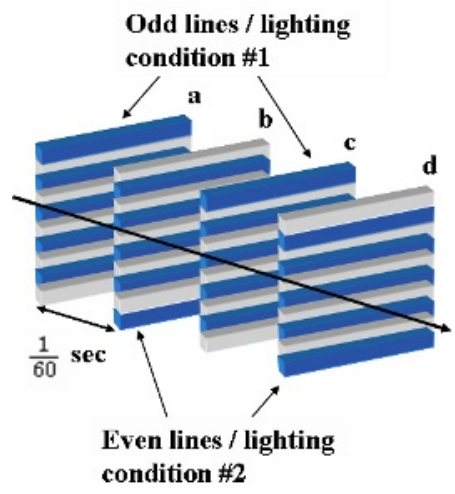

(a) Acquisition of video sequences

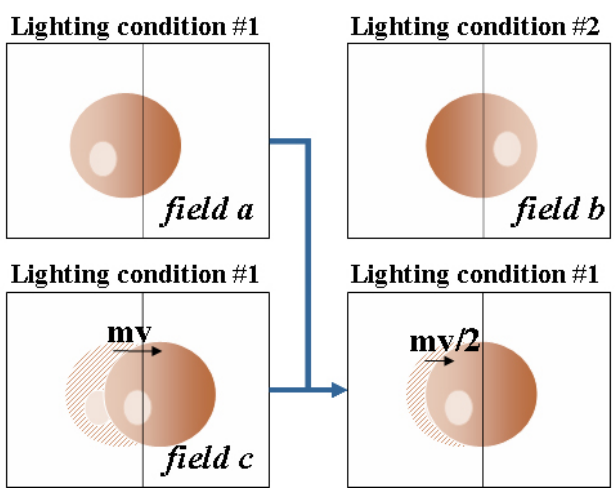

(b) Motion-compensated interpolation

Fig. 2. Computation of a pair of images for relighting a moving object using the motioncompensated interpolation. 
condition, a pair of images required for relighting a moving object are obtained. Figure 2 depicts the procedure of obtaining the required images.

A simple block matching algorihtm is used to estimate the motion of an object. In Figure 2, an image is created on which an object is located in the same position as the object in field $b$ by moving an object from the position of the object in field $a$ with a half of the motion vector of the object between field $a$ and $c$. More sophisticated algorithms would be necessary for dealing with complex motion and occlusion problems, which would be valuable for future work.

An additional line interpolation algorithm is employed because the image synthesized from field $a$ and $c$ has different even/odd lines from field $b$ as shown in Fig 2, We use a simple vertical averaging filter with the kernel of $(0.5,0,0.5)$. If the image includes a lot of high-frequency information, more sophisticated algorithms might be required.

\section{Experimental Results}

To obtain the video sequences used in the experiments, we captured a moving object in a darkroom. The strobe light was used which was synchronized with double frame-rate of a camera by a external trigger and thus the even and odd fields of video sequences have different lighting condition from each other. Figure 3 shows the experimental equipments used in our experiments. The images are at a resolution of 256 by 256 pixels and true-colored.

We take the procedure of Figure 4. From consecutive even and odd fields of the video sequences, a pair of synchronized images where an object is located at the same position but has different light condition from each other were first interpolated. Next, the specular and diffuse reflection were separated from the images. To relight an object, we finally applied a linear interpolation algorithm to the resulting diffuse reflection and a morphing algorithm to the resulting specular reflection, separately. The process was repeated on the field-by-field basis.

Figure 5 shows the results of computing the intermediate image from the two consecutive synthetic images using the motion-compensated interpolation

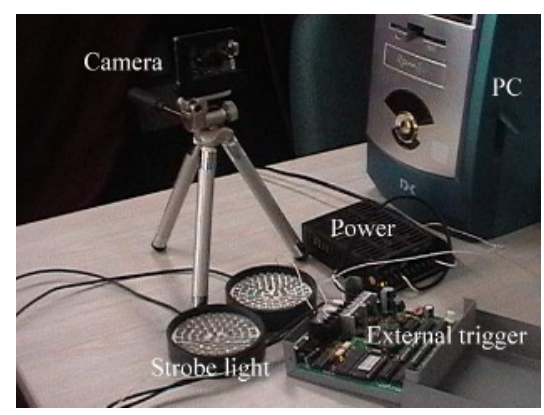

Fig. 3. Experimental equipments 


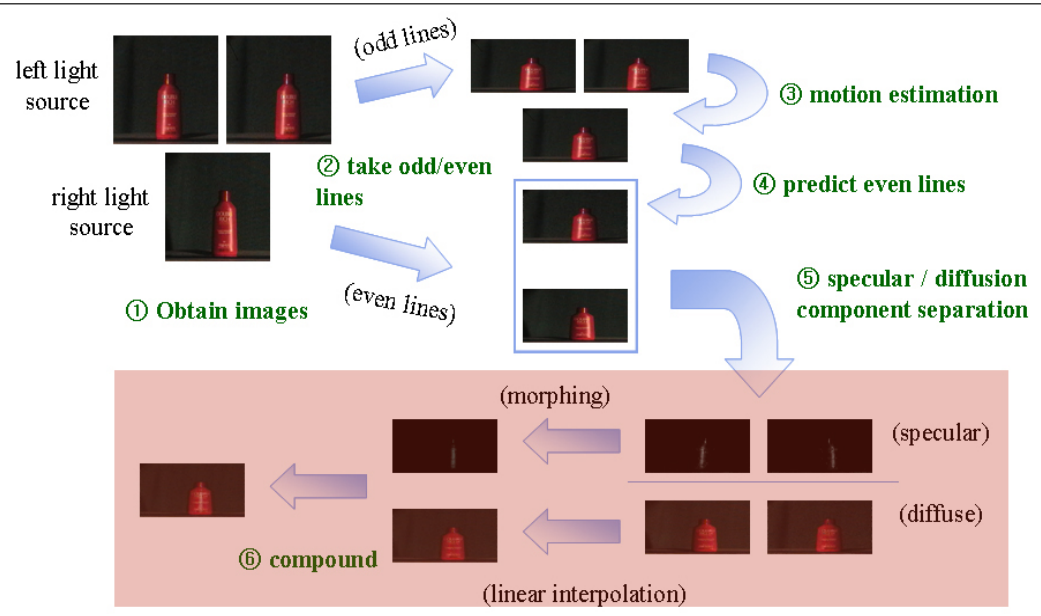

Fig. 4. Procudure for relighting a moving object. The shaded region depicts the process for relighting a static object

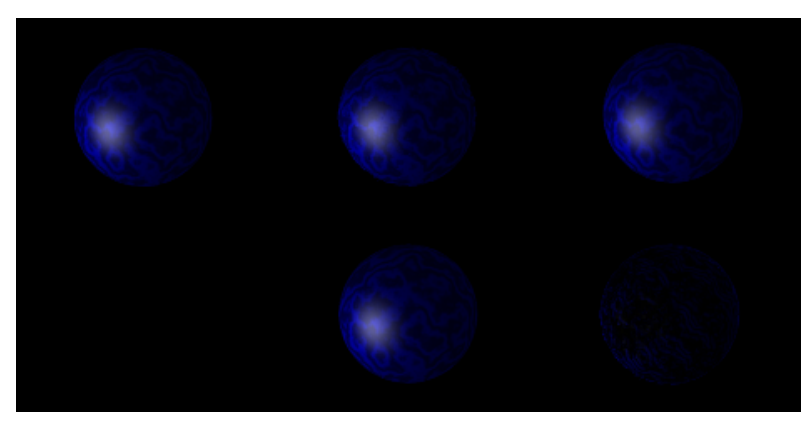

Fig. 5. Motion-compensated interpolation. Left-top: original n-th image. Right-top: original $(n+2)$-th image. Middle-top: the $(n+1)$-th computed image. Middle-bottom: ground truth image. Right-bottom: difference between the ground truth image and the $(\mathrm{n}+1)$-th computed image $(\mathrm{PSNR}=33.08 \mathrm{~dB})$.

algorithm which are explained in the previous section. The intermediate images were accurately computed $(\mathrm{PSNR}=33.08 \mathrm{~dB})$.

Figure 6 shows the results of relighting real and synthetic images based on the proposed relighting algorithm. In case of real images, the relit images were a little bit deteriorated by the effect of brightness saturation, shadow, incomplete specular reflection, and so on. However, the position and shape of synthesized specular reflection are very natural. The overall quality of the synthesized relit image is quite compelling when taking the fact that only the two images was used into the consideration.

To demonstrate the performance of the proposed strategy for a moving object, The result of relighting a moving object was compared with a ground-truth 

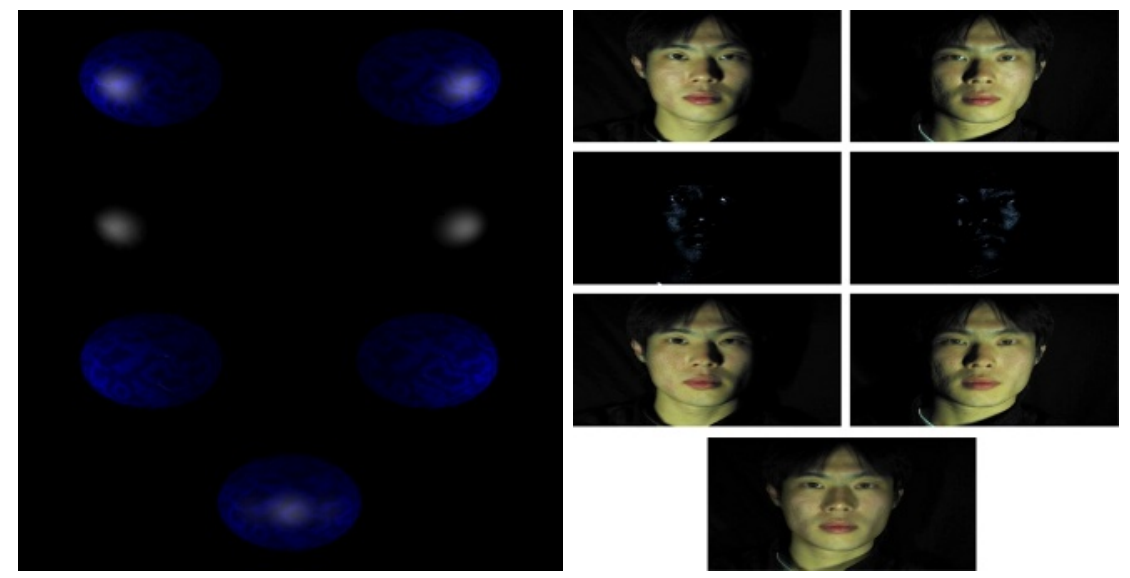

Fig. 6. Results of relighting a synthetic and real image based on the separation of diffuse and specular reflection. 1st-row images: synchronized images. 2nd-row images: the specular images computed from the synchronized images. 3rd-row images: the diffuse images computed from the synchronized images. 4th-row image: the relit images.

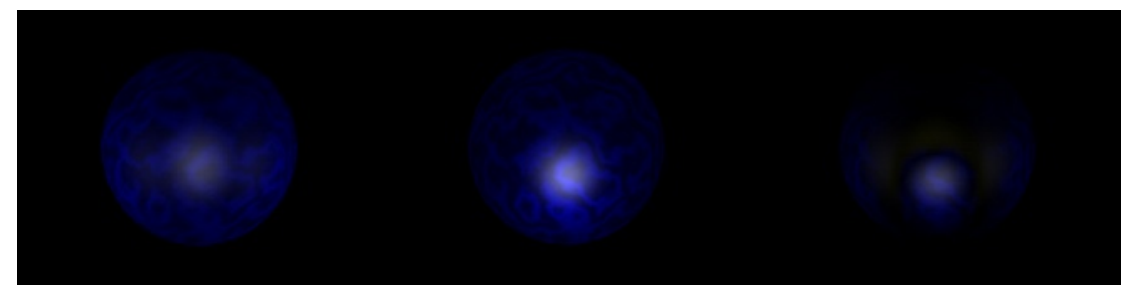

Fig. 7. Comparison of the relit image and a ground-truth image. (a) the result of relighting a moving object, (b) ground-truth image, (c) the difference of the two images $(\mathrm{PSNR}=27.87 \mathrm{~dB})$. The specular reflection of the relit image is visually compelling but a little darker than the specular reflection in the ground-truth image. It is assumed that the problem happens because the intensity of specular reflection is different according to its position.

image as shown in Figure 7 The relit image was a little bit blurred compared to a ground-truth image but the difference was quite small.

\section{Conclusion}

In this paper, we proposed a method of relighting a moving object based on the separation of specular and diffuse reflection. The method is an extension of the method for a static object [9]. Using controlled light sources and interlaced video sequence, we have successfully generated a pair of synchronized images with different lighting condition. Then, the conventional method for a static object was applied. The effectiveness of the proposed method was demonstrated through a variety of experimental results. 
The relit image was slightly different from a ground-truth image but visually convincing. In the future, interpolation algorithms suited for the proposed relighting method should be further explored. The method cannot fully cope with the change of shape of specular reflection between consecutive fields. More sophisticated methods towards resolving those problems should be investigated in the future.

\section{Acknowledgement}

This study was supported by a grant(02-PJ3-PG6-EV04-0003) of Ministry of Health and Welfare, Republic of Korea.

\section{References}

1. Fournier, A., Gunawan, S., Romanzin, C.: Common Illumination between Real and Computer Generated Scenes. Proc. of Graphics Interface (1993) 254-262

2. Gibson, S., Murta, A.: Interactive Rendering with Real-World Illumination. Proc. of Eurographics Workshop on Rendering (2000) 365-376

3. Loscos, C., Drettakis, G., Robert, L.: Interactive Virtual Relighting of Real Scenes. IEEE Transactions on Visualization and Computer Graphics (2000) 289-305

4. Kim, H.: Virtualizing Facial Images Considering Lighting Condition. MS thesis, Hanyang University, Seoul, Korea (2001)

5. Klinker, G.J., Shafer, S.A., Kanade, T.: The Measurement of Highlights in Color Images. International Journal of Computer Vision (1990) 7-32

6. Lin, S., Shum, H.Y.: Separation of Diffuse and Specular Reflection in Color Images. Proc. of CVPR (2001) 341-346

7. Wolff, L.B.: Using Polarization to Separate Reflection Components. Proc. of CVPR (1989) 363-369

8. Nayar, S.K., Fang, X., Boult, T.E.: Removal of Specularities Using Color and Polarization. Proc. of CVPR (1993) 583-590

9. Lee, B.-H.: Image-Based Relighting Using Separation of Specular and Diffuse Reflectance. MS thesis, Hanyang Univesity, Seoul, Korea (2004) (in Korean)

10. Lee, H.-C.: Method for Computing the Scene-Illuminant Chromaticity from Specular Highlights. Journal of the Optical Society of America A (1986) 1694-1699

11. Debevec, P., Hawkins, T., Tchou, C., Duiker, H.-P., Sarokin, W., Sagar, M.: Acquiring the Reflectance Field of a Human Face. Proc. of SIGGRAPH (2000) 145-156

12. Haeberli, P.: Synthetic Lighting for Photography. (1992) Available on http://www.sgi.com/grafica/synth/index.html

13. Wong, T.-T., Heng, P.-A., Or, S.-H, Ng, W.-Y.: Image-Based Rendering with Controllable Illumination. Proc. of Eurographics Workshop on Rendering (1997) 13-22

14. Shafer, S.A.: Using Color to Separate Reflection Components. Color Research and Applications (1985) 210-218 